\section{FRI0451 CONTINUOUS PRESENCE OF IGM ANTI- TOPOISOMERASE I ANTIBODIES INDICATES AN ONGOING IMMUNE RESPONSE IN SYSTEMIC SCLEROSIS}

M. Boonstra ${ }^{1}$, J.A. Bakker ${ }^{2}$, A. Grummels ${ }^{2}$, M.K. Ninaber ${ }^{3}$, N. Ajmone Marsan ${ }^{4}, \mathrm{H}$. U. Scherer ${ }^{1}$, R.E. Toes ${ }^{1}$, T.W. Huizinga ${ }^{5}$, J.K. de Vries-Bouwstra ${ }^{1} .{ }^{1}$ department of Rheumatology, ${ }^{2}$ department of Clinical Chemistry and Laboratory Medicine; ${ }^{3}$ department of Pulmonology; ${ }^{4}$ department of Cardiology, Leiden University Medical Center, ${ }^{5}$ department of Rheumatology, Leiden University Medical Center, Rheumatology, Leiden, Netherlands

Background: Small case-series of anti-topoisomerase I antibodies (ATA) in Systemic Sclerosis (SSc) show a highly varying immune response over time. IgA and IgG levels were shown to correlate with skin scores. One small study showed that increasing lgG levels can precede increasing skin scores. Thus far, detailed analysis of ATA characteristics with disease features in larger cohorts have not been performed.

Objectives: By taking advantage of our well described SSc cohort with annual follow-up data, we aimed to evaluate whether clinical heterogeneity within ATA + patients can be explained by ATA characteristics.

Methods: ATA IgG, IgM and IgA levels were assessed in consecutive serum samples of baseline ATA-lgG + patients from the Leiden SSc cohort. Disease progression during the first year of follow-up was defined as increase of modified Rodnan Skin Score (mRSS) with $\geq 5$ points, progression of pulmonary involvement $(-\leq 10 \%$ of predicted forced vital capacity [FVC] or diffusion capacity of the lung [DLCO]), development of digital ulcers, renal crisis, pulmonary arterial hypertension and/or mortality. Here, we present data on the association between the presence of ATA-IgM and disease progression.

Results: In total 344 samples of 102 ATA +patients were measured. Baseline and follow-up samples were available from 70 patients. Median follow-up was 3.7 years (range 0.9-7.4 years). At baseline 42/70 patients were positive for ATA IgM and 69/70 patients for ATA IgA (table 1). Strikingly, while clinical characteristics did not differ, mean ATA-IgM at baseline was higher in disease progressors (table 2). The possible relevance of an ATA-IgM response for disease progression was confirmed by the observation that of those patients positive for ATA-IgM both at baseline and at FU, $59 \%$ of cases showed disease progression, as compared to $15 \%$ of patients negative for ATA IgM at both time points $(p=0.02)$.

Abstract FRI0451 - Table 1. Changes in presence and levels of ATA isotypes in paired serum samples

\begin{tabular}{|c|c|c|c|c|c|c|c|}
\hline & \multicolumn{4}{|c|}{$\begin{array}{l}\text { ATA isotype status at baseline/ } \\
\text { follow-up }\end{array}$} & \multicolumn{3}{|c|}{ median ATA level (aU/mL[IQR]) } \\
\hline & $+/+$ & \pm & $-/-$ & $-1+$ & baseline & follow-up & $\mathrm{p}$ \\
\hline $\lg G$ & 66 & 4 & - & - & $454(193-876)$ & $283(79-647)$ & $<0.01$ \\
\hline $\lg M$ & 29 & 13 & 25 & 3 & $627(271-2081)$ & $348(167-1315)$ & 0.06 \\
\hline $\operatorname{Ig} A$ & 65 & 4 & 1 & 0 & $2832(812-8069)$ & $2210(645-7593)$ & 0.01 \\
\hline
\end{tabular}

Abstract FRI0451 - Table 2. Baseline characteristics of SSc patients with and without disease progression during the first year of follow-up

\begin{tabular}{|c|c|c|c|}
\hline & $\begin{array}{l}\text { progressors } \\
(n=25)\end{array}$ & $\begin{array}{c}\text { non- } \\
\text { progressors } \\
(n=36)\end{array}$ & p \\
\hline female, $n(\%)$ & $18(72)$ & $28(78)$ & 0.61 \\
\hline age, mean $[y r s.] \pm S D$ & $55 \pm 16$ & $50 \pm 15$ & 0.15 \\
\hline $\begin{array}{l}\text { time since onset first non-Raynaud symptom, } \\
\text { median [yrs.] (IQR) }\end{array}$ & $4(1-8)$ & $3(1-12)$ & 0.75 \\
\hline mRSS, median (IQR) & $4(3-8)$ & $5(2-13)$ & 0.94 \\
\hline DLCO, mean $[\%$ of predicted] $\pm S D$ & $69(56-81)$ & $63(49-75)$ & 0.17 \\
\hline ATA lgG level, median [aU/mL](IQR) & $\begin{array}{l}636(301- \\
1127)\end{array}$ & $312(111-900)$ & 0.09 \\
\hline ATA IgM level, median [aU/mL](IQR) & $\begin{array}{l}933(533- \\
3122)\end{array}$ & $447(215-1293)$ & 0.03 \\
\hline ATA IgA level, median [aU/mL](IQR) & $\begin{array}{c}5217(2003- \\
14187)\end{array}$ & $\begin{array}{l}2202(513- \\
6240)\end{array}$ & 0.07 \\
\hline
\end{tabular}

Conclusions: The presence of ATA-IgM at baseline and at follow-up and its association with disease course suggests that the ATA response in SSc patients is an ongoing process that possibly explains the heterogenic disease course of ATA + patients over time.

Disclosure of Interest: None declared

DOI: 10.1136/annrheumdis-2018-eular.6267

\section{FRI0452 \\ NAILFOLD CAPILLAROSCOPY IN ANTISYNTHETASE SYNDROME (NASCAR): RESULTS OF A MULTICENTER, INTERNATIONAL STUDY OF THE AMERICAN AND EUROPEAN NETWORK OF ANTISYNTHETASE SYNDROME (AENEAS)}

M. Sebastiani ${ }^{1}$, K. Triantafyllias ${ }^{2}$, A. Manfredi ${ }^{1}$, M.A. González-Gay ${ }^{3}, N$. PalmouFontana $^{3}$, G. Cassone ${ }^{1}$, U. Drott ${ }^{4}$, C. Delbrück ${ }^{4}, J$. Rojas-Serrano ${ }^{5}, C$. Bertolazzi $^{5}$, L. Nuño ${ }^{6}$, M. Giannini ${ }^{7}$, F. lannone ${ }^{7}$, E.F. Vicente ${ }^{8}$, S. Castañeda ${ }^{8}$, A. SelvaO'Callaghan $^{9}$, E. Trallero Araguas ${ }^{9}$, G. Emmi ${ }^{10}$, A. Iuliano ${ }^{11}$, J. Bauhammer ${ }^{12}$, N. Miehle ${ }^{12}$, S. Parisi ${ }^{13}$, L. Cavagna ${ }^{14}$, V. Codullo ${ }^{14}$, C.M. Montecucco ${ }^{14}$, F.J. Lopez Longo ${ }^{15}$,J. Martínez-Barrio ${ }^{15}$, J.C. Nieto-González ${ }^{15}$, S. Vichi ${ }^{16}$, M. Confalonieri ${ }^{17}$, R. Bergner ${ }^{18}$, A. Sulli ${ }^{19}$, F. Bonella ${ }^{20}$, F. Furini ${ }^{21}$, C.A. Scirè ${ }^{21}$, C. Specker ${ }^{22}$, S. Barsotti ${ }^{23}$, R. Neri ${ }^{23}$, J. Weinmann-Menke ${ }^{24}$, A. Schwarting ${ }^{24}$, V. Smith ${ }^{25}$, M. Cutolo ${ }^{26}$ on behalf of NASCAR Study Group. ${ }^{1}$ Rheumatology Unit, University of Modena and Reggio Emilia, Modena, Italy; ${ }^{2}$ Rheumatology Unit, ACURA Rheumatology Center, Bad Kreuznach, Germany; ${ }^{3}$ Rheumatology Unit, University of Cantabria, Santander, Spain; ${ }^{4}$ Rheumatology Unit, University Hospital of Frankfurt, Frankfurt, Germany; ${ }^{5}$ Interstitial Lung disease and Rheumatology Unit, Instituto Nacional de Enfermedades Respiratorias, Mexico City, Mexico; ${ }^{6}$ Servicio de Reumatología, Hospital Universitario La Paz, Madrid, Spain; ${ }^{7}$ Rheumatology Unit, University of Bari, Bari, Italy; ${ }^{8}$ Rheumatology Department, Hospital Universitario de la Princesa, Madrid; ${ }^{9}$ Servicio de Medicina Interna, Universidad Autonoma de Barcelona, Barcelona, Spain; ${ }^{10}$ Department of Experimental and Clinical Medicine, University of Florence, Florence; ${ }^{11}$ Rheumatology Unit, Ospedale San Camillo-Forlanini, Roma, Italy; ${ }^{12}$ Rheumatology Unit, ACURA Centre for Rheumatic Diseases, Baden-Baden, Germany; ${ }^{13}$ Rheumatology Department, Città Della Salute e della Scienza, Torino; ${ }^{14}$ Rheumatology Unit, University and IRCCS Policlinico S. Matteo Foudation, Pavia, Italy; ${ }^{15}$ Servicio de Reumatología, Hospital General Universitario Gregorio Marañón, Madrid, Spain; ${ }^{16}$ Dermatologic Clinic, University Hospital of Trieste; ${ }^{17}$ Department of Pneumology and Respiratory Intermediate Care Unit, University Hospital of Cattinara, Trieste, Italy; ${ }^{18}$ Medizinische Klinik A, Klinikum der Stadt, Ludwigshafen, Germany; ${ }^{19}$ Research Laboratory and Academic Division of Clinical Rheumatology, University of Genova, Genova, Italy; ${ }^{20}$ Interstitial and Rare Lung Disease Unit, University of Duisburg-Essen, Essen, Germany; ${ }^{21}$ Rheumatology Unit, University of Ferrara, Ferrara, Italy; ${ }^{22}$ Department for Rheumatology and Clinical Immunology, St. Josef Krankenhaus, University Clinic, Essen, Germany; ${ }^{23}$ Rheumatology Unit, Department of Clinical and Experimental Medicine, Pisa, Italy; ${ }^{24}$ Department of Internal Medicine, University Hospital Johannes-Gutenberg, Mainz, Germany; ${ }^{25}$ Rheumatology Unit, Ghent University Hospital, Ghent, Belgium; ${ }^{26}$ Rheumatology Unit, University of Genova, Genova, Italy

Background: Antisynthetase syndrome (ASSD) is an autoimmune disease char acterised by the clinical triad arthritis, myositis, and interstitial lung disease (ILD). Despite Raynaud's phenomenon (RP) is another typical feature of ASSD, nailfold videocapillaroscopy (NVC) assessment of these patients has been only sporadically described, without the elucidating data for clinicians.

Objectives: To describe NVC features of ASSD patients and to investigate possible correlations with clinical and serological features of the disease.

Methods: We retrospectively analysed NVC images of 190 ASSD patients (females/males 3.76, mean age $49.7 \pm 12.8$ years, mean disease duration 51.2 \pm 71.4 months, 133 anti-Jo-1 and 57 non-anti-Jo- 1 positive patients). For each patient, we examined number of capillaries, giant capillaries, micro-haemorrhages, avascular areas, ramified capillaries, and the presence of scleroderma (SSc) patterns. Finally, we correlated NVC features with clinical and serological findings of ASSD patients.

Results: NVC abnormalities were observed in $62.1 \%$ of AASD patients compared with $29.3 \%$ of a group of 75 patients with primary Raynaud's phenomenon $(p<0.001)$. A SSc-like pattern was detected in $67(35.3 \%)$ patients and it was associated with anti-Jo-1 antibodies $(\mathrm{p}=0.002)$ and also with a longer disease duration $(p=0.004)$. Interestingly, there was no significant correlation between the presence of SSc-like pattern and RP, and only $47 \%$ of patients with SSc-like pattern had RP.

Conclusions: NVC abnormalities are commonly observed in ASSD, independently to the occurrence of RP. The presence of a SSc-like pattern should let to identify a more defined ASSD subtype and prospective studies could confirm the association with clinical and serological features of ASSD.

Disclosure of Interest: None declared

DOI: 10.1136/annrheumdis-2018-eular.2859 